\title{
Exact metric for the exterior of a global string in the Brans-Dicke theory
}

\author{
B. Boisseau, and B. Linet ${ }^{\dagger}$ \\ Laboratoire de Mathématiques et Physique Théorique \\ CNRS/UPRES-A 6083, Université François Rabelais \\ Faculté des Sciences et Techniques \\ Parc de Grandmont 37200 TOURS, France
}

\begin{abstract}
We determine in closed form the general static solution with cylindrical symmetry to the Brans-Dicke equations for an energy-momentum tensor corresponding to the one of the straight $\mathrm{U}(1)$ global string outside the core radius assuming that the Goldstone boson field takes its asymptotic value.
\end{abstract}

\section{Introduction}

Topological defects could be produced at a phase transition in the early universe [1], 2]. Their nature depends on the symmetry broken in the field theory under consideration. A class of topological defects are the global defects as the global strings which are not finite energy. So, a static, straight U(1) global string in general relativity has a metric which is not asymptotically Minkowskian [3, 田, 5. 6]. Indeed, the spacetime has necessarily a physical singularity at a finite proper distance of the axis [1] giving constraints on the abundance of the global strings in the early universe [7]. The explicit metric outside the core radius within the approximate theory in which the Goldstone boson field takes its asymptotic value has been obtained by Cohen and Kaplan [0] and it presents a curvature singularity at a finite proper distance.

It is widely accepted that a gravitational scalar field, beside the metric of the spacetime, must exist in the framework of the present unified theories. These scalar-tensor theories of gravitation take their importance in the early universe where it is expected that the coupling to matter of the scalar field would be as same order as the one of metric althought the scalar coupling is negligeable in the present time. Now topological defects are produced

\footnotetext{
*E-mail : boisseau@celfi.phys.univ-tours.fr

${ }^{\dagger}$ E-mail : linet@celfi.phys.univ-tours.fr
} 
during vacuum phase transitions in the early universe, therefore several authors studied the static solutions generated for instance by a straight $\mathrm{U}(1)$ gauge string in the Brans-Dicke theory [8, 9], in the scalar-tensor theories with matter minimally coupled [10] or in the dilaton theories [11]. Of course the scalar field is supposed massless because in the massive case the theory is pratically general relativity for distance much larger than the range of the scalar field.

Recently, Sen et al [12] studied the static, straight U(1) global string in the Brans-Dicke theory but they did not arrive to determine in closed form the general solution outside the core radius of the straight $\mathrm{U}(1)$ global string. Also, we take up again the problem of the determination of the exact solution outside the core radius in the case where the Goldstone boson field takes its asymptotic value.

The gravitational field variables of the Brans-Dicke theory are the metric $\hat{g}_{\mu \nu}$ of the spacetime and a scalar field $\hat{\phi}$. The matter is minimally coupled to $\hat{g}_{\mu \nu}$ and its energymomentum tensor $\hat{T}_{\nu}^{\mu}$ is conserved. Nevertheless, it is now well-known that it is more convenient to use a non-physical metric $g_{\mu \nu}$ and a new scalar $\phi$ defined by

$$
\hat{g}_{\mu \nu}=\exp (2 \alpha \phi) g_{\mu \nu} \quad \text { and } \quad \hat{\phi}=\frac{1}{\mathcal{G}} \exp (-2 \alpha \phi)
$$

where the gravitational coupling constant $\mathcal{G}$ can be related to the Newtonian constant. The parameter $\alpha$ in (何) has the value

$$
\alpha^{2}=\frac{1}{2 \omega+3}
$$

in terms of the usual Brans-Dicke parameter $\omega$. We also introduce a non-physical source $T_{\mu \nu}$ defined by

$$
T_{\mu \nu}=\exp (2 \alpha \phi) \hat{T}_{\mu \nu} .
$$

We describe the straight $\mathrm{U}(1)$ global string by a static, cylindrically symmetric spacetime. Consequently, we can write the non-physical metric in the form

$$
d s^{2}=d \rho^{2}+g_{2}(\rho) d z^{2}+g_{3}(\rho) d \varphi^{2}-g_{4}(\rho) d t^{2}
$$

in the coordinate system $(\rho, z, \varphi, t)$ with $\rho>0$ and $0 \leq \varphi<2 \pi$ where the functions $g_{2}, g_{3}$ and $g_{4}$ are strictly positive. The scalar field depends only on $\rho$. Under our assumptions, the form of the energy-momentum tensor $\hat{T}_{\nu}^{\mu}$ outside the core radius of the $\mathrm{U}(1)$ global string yields

$$
T_{\rho}^{\rho}=T_{z}^{z}=T_{t}^{t}=-T_{\varphi}^{\varphi}=-\sigma(\rho)
$$

where the strictly positive function $\sigma$ is to be determined for $\rho>\rho_{C}, \rho_{C}$ being the core radius. As noticed by Gibbons et al [13], form (田) corresponds also to a $\sigma$-model with vanishing potential and having a target space with closed geodesics.

The purpose of this work is to give the general expression of static metrics with cylindrical symmetry and the scalar field which are the solutions to the Brans-Dicke equations 
with a source having the algebraic form (4). In the particular case of a straight U(1) global string, metric (3) must exhibit the boost invariance, i.e. $g_{2}=g_{4}$.

The plan of the work is as follows. In Section 2, we give the basic equations of our problem which are to be solved. The explicit solutions are obtained in Section 3. We discuss in Section 4 the singularities of the solutions and the existence of black hole solutions. We add in Section 5 some concluding remarks.

\section{Gravitational field equations}

In terms of the non-physical metric $g_{\mu \nu}$ and the scalar field $\phi$ introduced by relations (1), the Brans-Dicke equations are

$$
\begin{gathered}
R_{\mu \nu}=2 \partial_{\mu} \phi \partial_{\nu} \phi+8 \pi \mathcal{G}\left(T_{\mu \nu}-\frac{1}{2} T g_{\mu \nu}\right), \\
\square \phi=-4 \pi \mathcal{G} \alpha T
\end{gathered}
$$

where the source is the non-physical energy-momentum tensor (2). We derive from (5) and (6) that

$$
\nabla_{\mu} T_{\nu}^{\mu}=\alpha T \partial_{\nu} \phi
$$

We note that equation (5) can be rewritten

$$
G_{\mu \nu}=R_{\mu \nu}-\frac{1}{2} R g_{\mu \nu}=2 \partial_{\mu} \phi \partial_{\nu} \phi-g_{\mu \nu} g^{\gamma \delta} \partial_{\gamma} \phi \partial_{\delta} \phi+8 \pi \mathcal{G} T_{\mu \nu}
$$

For metrics (3) with source (4), equation (7) reduces to

$$
\frac{d \sigma}{d \rho}+\frac{\sigma}{g_{3}} \frac{d g_{3}}{d \rho}=2 \alpha \sigma \frac{d \phi}{d \rho}
$$

whose the general solution has the form

$$
\sigma(\rho)=\frac{\sigma_{0}}{8 \pi \mathcal{G}} \frac{\exp (2 \alpha \phi(\rho))}{g_{3}(\rho)}
$$

where $\sigma_{0}$ is an arbitrary positive constant.

We are now in a position to write down the gravitational field equations. In equation (5), the components $R_{z}^{z}, R_{\varphi}^{\varphi}$ and $R_{t}^{t}$ of the Ricci tensor give firstly

$$
\begin{gathered}
\frac{d}{d \rho}\left(\frac{u}{g_{2}} \frac{d g_{2}}{d \rho}\right)=0 \\
\frac{d}{d \rho}\left(\frac{u}{g_{3}} \frac{d g_{3}}{d \rho}\right)=-4 \sigma_{0} \frac{u \exp (2 \alpha \phi)}{g_{3}} \\
\frac{d}{d \rho}\left(\frac{u}{g_{4}} \frac{d g_{4}}{d \rho}\right)=0
\end{gathered}
$$


where $u$ denotes the square root of the determinant, $u^{2}=g_{2} g_{3} g_{4}$. Secondly, the scalar equation (6) is written as

$$
\frac{d}{d \rho}\left(u \frac{d \phi}{d \rho}\right)=\alpha \sigma_{0} \frac{u \exp (2 \alpha \phi)}{g_{3}} .
$$

In equation (8), the component $G_{\rho}^{\rho}$ of the Einstein tensor gives a constraint equation

$$
\frac{1}{g_{2} g_{3}} \frac{d g_{2}}{d \rho} \frac{d g_{3}}{d \rho}+\frac{1}{g_{3} g_{4}} \frac{d g_{3}}{d \rho} \frac{d g_{4}}{d \rho}+\frac{1}{g_{4} g_{2}} \frac{d g_{4}}{d \rho} \frac{d g_{2}}{d \rho}=-4 \sigma_{0} \frac{\exp (2 \alpha \phi)}{g_{3}}+4\left(\frac{d \phi}{d \rho}\right)^{2} \text {. }
$$

We have a system of five differential equations for $g_{2}, g_{3}, g_{4}$ and $\phi$ which are compatible since we have taken a $T_{\mu \nu}$ satisfying identically the integrability condition (7).

In order to solve these equations we introduce a new radial coordinate $r$ related to $\rho$ by

$$
u(\rho) \frac{d r}{d \rho}=1 .
$$

We have $-\infty<r<\infty$ when $0<\rho<\infty$ since a priori the integral of $1 / u$ diverges as $\rho \rightarrow 0$. In this coordinate system metric (3) has the form

$$
d s^{2}=g_{2}(r) g_{3}(r) g_{4}(r) d r^{2}+g_{2}(r) d z^{2}+g_{3}(r) d \varphi^{2}-g_{4}(r) d t^{2} .
$$

Of course, the energy-momentum tensor keeps form (4). The gravitational field equations (10-14) become

$$
\begin{gathered}
\frac{d}{d r}\left(\frac{1}{g_{2}} \frac{d g_{2}}{d r}\right)=0 \\
\frac{d}{d r}\left(\frac{1}{g_{3}} \frac{d g_{3}}{d r}\right)=-4 \sigma_{0} g_{2} g_{4} \exp (2 \alpha \phi), \\
\frac{d}{d r}\left(\frac{1}{g_{4}} \frac{d g_{4}}{d r}\right)=0 \\
\frac{d^{2} \phi}{d r^{2}}=\alpha \sigma_{0} g_{2} g_{4} \exp (2 \alpha \phi), \\
\frac{1}{g_{2} g_{3}} \frac{d g_{2}}{d r} \frac{d g_{3}}{d r}+\frac{1}{g_{3} g_{4}} \frac{d g_{3}}{d r} \frac{d g_{4}}{d r}+\frac{1}{g_{4} g_{2}} \frac{d g_{4}}{d r} \frac{d g_{2}}{d r}=-4 \sigma_{0} g_{2} g_{4} \exp (2 \alpha \phi)+4\left(\frac{d \phi}{d r}\right)^{2} .
\end{gathered}
$$

\section{Explicit determination of the solutions}

Equations (17) and (19) have the obvious solutions

$$
g_{2}(r)=g_{2}^{0} \exp \left(K_{2} r\right) \quad \text { and } \quad g_{4}(r)=g_{4}^{0} \exp \left(K_{4} r\right)
$$

where $g_{2}^{0}, K_{2}, g_{4}^{0}$ and $K_{4}$ are arbitrary constants. By combining equations (18) and (20) we obtain the relation

$$
g_{3}(r)=g_{3}^{0} \exp \left(K_{3} r\right) \exp \left(-\frac{4}{\alpha} \phi\right)
$$

where $g_{3}^{0}$ and $K_{3}$ are arbitrary constants. Hereafter we denote $g_{3}$ by $g$. We have now to distinguish two cases. 


\subsection{Case $K_{2}=0$ and $K_{4}=0$}

In the case where $K_{2}=0$ and $K_{4}=0$, metric (16) reduces to

$$
d s^{2}=g(r) d r^{2}+d z^{2}+g(r) d \varphi^{2}-d t^{2}
$$

in rescaled coordinates $r, z$ and $t$. It remains three equations of system (17-21). Equation (21) leads to

$$
\frac{d \phi}{d r}= \pm \sqrt{\sigma_{0}} \exp (\alpha \phi)
$$

and from this equation (20) is automatically satisfied. We can integrate equation (25) and we get the expression of the scalar field

$$
\exp (2 \alpha \phi)=\frac{1}{\alpha^{2} \sigma_{0}(r-k)^{2}}
$$

where $k$ is an arbitrary constant. Taking into account (23) we now obtain the components of metric (24)

$$
g(r)=g^{0} \exp (K r)|r-k|^{4 / \alpha^{2}}
$$

\subsection{Case $K_{2} \neq 0$ or $K_{4} \neq 0$}

When $K_{2} \neq 0$ or $K_{4} \neq 0$, it is always possible to write $K_{2}=\mu(1-w)$ and $K_{4}=\mu(1+w)$ for specific constants $\mu$ and $w, \mu \neq 0$. We then introduce a dimensionless coordinate $x$ by setting

$$
x=\exp (\mu r)
$$

where $0<x<\infty$. If $\mu>0$ then the coordinate $x$ is increasing with $r$. In this coordinate system metric (3) has the form

$$
d s^{2}=\frac{1}{\mu^{2}} g_{2}^{0} g_{4}^{0} g_{3}(x) d x^{2}+g_{2}^{0} x^{1-w} d z^{2}+g_{3}(x) d \varphi^{2}-g_{4}^{0} x^{1+w} d t^{2} .
$$

By a rescaling of the coordinates $x, z$ and $t$, we can put this metric in the following form

$$
d s^{2}=g(x) d x^{2}+x^{1-w} d z^{2}+g(x) d \varphi^{2}-x^{1+w} d t^{2}
$$

The energy-momentum tensor keeps form (四) and $\sigma$ is given by (9).

It is convenient to write directly the field equations (5) and (6) for metric (29); we thus obtain

$$
\begin{gathered}
\frac{1}{x^{2}}-\frac{w^{2}}{x^{2}}+\frac{g^{\prime}}{x g}+\frac{\left(g^{\prime}\right)^{2}}{g^{2}}-\frac{g^{\prime \prime}}{g}=4\left(\phi^{\prime}\right)^{2}, \\
\frac{\left(g^{\prime}\right)^{2}}{g^{2}}-\frac{g^{\prime \prime}}{g}-\frac{g^{\prime}}{x g}=4 \sigma_{0} \exp (2 \alpha \phi), \\
\frac{1}{x}\left(x \phi^{\prime}\right)^{\prime}=\alpha \sigma_{0} \exp (2 \alpha \phi),
\end{gathered}
$$


the other components being identically verified. Moreover relation (23) is now written as

$$
g(x)=g^{0} x^{c} \exp \left(-\frac{4}{\alpha} \phi\right)
$$

where $c$ are an arbitrary constant.

We now introduce the function $y(x)$ defined by $y(x)=2 \alpha \phi$. Taking into account (33), the system of differential equation 30 32 reduces to

$$
\begin{gathered}
2 x^{2} y^{\prime \prime}-2 x y^{\prime}-x^{2} y^{\prime 2}+\alpha^{2}\left(1-w^{2}+2 c\right)=0, \\
y^{\prime \prime}+\frac{1}{x} y^{\prime}=2 \alpha^{2} \sigma_{0} \exp y
\end{gathered}
$$

for the unknowm function $y$. In the appendix, we give the explicit expression of the common solutions to the differential equations (34) and (35) by setting $C=\alpha^{2}\left(1-w^{2}+2 c\right)$.

To summarise this, the desired metric (29), for a given $w$ and $g^{0}$, and the scalar field $\phi$ depends on three constants $C_{1}, C_{2}$ and $n$ because we express $c$ in terms of $n$ and $w$. A convenient classification of the solutions is to use the sign of $n$; we obtain thereby for $n>0$

$$
\begin{aligned}
& g(x)=g^{0} x^{\left(2 / \alpha^{2}-1 / 2+w^{2} / 2+2 n^{2} / \alpha^{2}\right)}[|Z(x)|]^{4 / \alpha^{2}} \\
& \phi(x)=-\frac{1}{\alpha}(\ln x+\ln |Z(x)|) \\
& \text { with } \quad Z(x)=C_{1} x^{n}+C_{2} x^{-n} \quad C_{1} C_{2}<0,
\end{aligned}
$$

for $n=0$

$$
\begin{aligned}
& g(x)=g^{0} x^{\left(2 / \alpha^{2}-1 / 2+w^{2} / 2\right)}[|Z(x)|]^{4 / \alpha^{2}} \\
& \phi(x)=-\frac{1}{\alpha}(\ln x+\ln |Z(x)|) \\
& \text { with } \quad Z(x)=C_{1}+C_{2} \ln x \quad C_{2} \neq 0
\end{aligned}
$$

and for $n<0$

$$
\begin{aligned}
& g(x)=g^{0} x^{\left(2 / \alpha^{2}-1 / 2+w^{2} / 2-2 n^{2} / \alpha^{2}\right)}[|Z(x)|]^{4 / \alpha^{2}} \\
& \phi(x)=-\frac{1}{\alpha}(\ln x+\ln |Z(x)|) \\
& \text { with } \quad Z(x)=C_{1} \sin (n \ln x)+C_{2} \cos (n \ln x) \quad C_{1} \neq 0 \quad \text { or } \quad C_{2} \neq 0 .
\end{aligned}
$$

The strictly positive value of $\sigma_{0}$ characterizing the energy-momentum tensor (9) is given in the appendix by relations (51) in terms of the constants $C_{1}, C_{2}$ and $n$ appearing in solutions $(36-38)$.

\section{Singularities of the solutions and black hole cases}




\subsection{Case $K_{2}=0$ and $K_{4}=0$}

We write down the physical metric (11) associated with metric (24) where the function $g$ is given by (27)

$$
d \hat{s}^{2}=\frac{g^{0}}{\alpha^{2} \sigma_{0}}|r-k|^{4 / \alpha^{2}-2} \exp (K r)\left(d r^{2}+d \varphi^{2}\right)+\frac{1}{\alpha^{2} \sigma_{0}(r-k)^{2}}\left(d z^{2}-d t^{2}\right)
$$

with $-\infty<r<\infty$ in principle. It is obvious that the Riemann tensor of metric (39) diverges at $r=k$. So, there exists two intervals of definition of the metric : $-\infty<r<k$ and $k<r<\infty$. The point $r=k$ is at a finite proper distance in the two domains $r<k$ and $r>k$ since the values of the proper radial coordinate, respectively given by the integrals

$$
\int^{r}(-r+k)^{2 / \alpha^{2}-1} \exp (K r / 2) d r \text { and } \int_{r}(r-k)^{2 / \alpha^{2}-1} \exp (K r / 2) d r
$$

are finite as $r \rightarrow k$. We see from (26) that the scalar field is also singular at $r=k$.

\subsection{Case $K_{2} \neq 0$ or $K_{4} \neq 0$}

In this case the physical metric (11) associated with metric (29) has the form

$$
d \hat{s}^{2}=\frac{g(x)}{x^{2} Z^{2}(x)}\left(d x^{2}+d \varphi^{2}\right)+\frac{x^{-1-w}}{Z^{2}(x)} d z^{2}-\frac{x^{-1+w}}{Z^{2}(x)} d t^{2}
$$

where the functions $Z$ and $g$ are given in (36-38) with in principle $0<x<\infty$. It is clear from the expression of the components of metric (40) that the zeros of the function $Z$ are the singularities of the Riemann tensor. They are at finite proper distances. The scalar field is also singular at the zeros of the function $Z$.

The case $x=0$ yields a singularity for metric (40) except if $x=0$ is an horizon. This eventuality occurs only possible for solution (36) when $-1+w+2 n>0$ since the component $g_{t t}$ is proportional to $x^{-1+w+2 n}$ as $x \rightarrow 0$. To obtain a black hole, we must have that the components $g_{z z}$ and $g_{\varphi \varphi}$ remain constant as $x \rightarrow 0$. Therefore we require

$$
w=2 n-1 \quad \text { and } 2 / \alpha^{2}-1 / 2+w^{2} / 2+2 n / \alpha^{2}-2-4 n / \alpha^{2}+2 n=0 .
$$

As a consequence, $g_{t t}$ is proportional to $x^{4 n-2}$ as $x \rightarrow 0$. We now perform the following change of radial coordinate

$$
R(x)=\frac{\sqrt{g(x)}}{x Z(x)} .
$$

Taking into account the previous relations, we find that the components $g_{R R}$ is proportional to $x^{-4 n+2}$ as $x \rightarrow 0$. Now there exists certainly a coordinate system in which the components of the metric are regular at $x=0$, therefore we obtain a family of black hole metrics (40) when the constants verify relations (41). The situation is similar in general relativity where a black hole metric exists for $w=1$ [14]. However, the scalar field is only regular at $x=0$ for $n=1 / 2$. Also, we have not really a solution to Brans-Dicke equations representing a black hole. 


\section{Conclusion}

We have explicitly found the general static solution with cylindrical symmetry to the Brans-Dicke equations with a source having the algebraic form (四). There are two classes of solutions: metrics (39) and metrics (40).

The general static metric describing a straight global string outside the core radius is obtained by requiring that $g_{2}=g_{4}$. We write down the physical metric (11) for the two classes. Firstly, we have directly metric (39)

$$
\begin{aligned}
& d \hat{s}^{2}=\frac{g^{0}}{\alpha^{2} \sigma_{0}}|r-k|^{4 / \alpha^{2}-2} \exp (K r)\left(d r^{2}+d \varphi^{2}\right)+\frac{1}{\alpha^{2} \sigma_{0}(r-k)^{2}}\left(d z^{2}-d t^{2}\right), \\
& \hat{\phi}=\frac{\alpha^{2} \sigma_{0}}{\mathcal{G}}(r-k)^{2} .
\end{aligned}
$$

This form of metric does not exist in general relativity. Secondly, by setting $w=0$ in metric (40) we get

$$
\begin{aligned}
& d \hat{s}^{2}=\frac{1}{x^{2} Z^{2}(x)}\left[g(x)\left(d x^{2}+d \varphi^{2}\right)+x\left(d z^{2}-d t^{2}\right)\right], \\
& \hat{\phi}=\frac{1}{\mathcal{G}} x^{2} Z^{2}(x) .
\end{aligned}
$$

where the functions $g$ and $Z$ are given in (36-38) with $w=0$. We have proved that the point $x=0$ and the zeros of the function $Z$ are physical singularities of the solutions in the Brans-Dicke theory. We have not touched upon the question concerning the matching of these solutions with the asymptotic solutions describing a straight U(1) global string.

\section{Appendix}

Following Kamke [15] p. 562, it is possible to find the common solutions to the following system of differential equations

$$
\begin{gathered}
y^{\prime \prime}+\frac{1}{x} y^{\prime}=2 \alpha^{2} \sigma_{0} \exp y, \\
2 x^{2} y^{\prime \prime}-2 x y^{\prime}-x^{2} y^{\prime 2}+C=0
\end{gathered}
$$

where $C$ is a constant. We firstly solve equation (45). By means of the change of function

$$
y(x)=-2 \ln x-2 \ln |Z(x)|,
$$

we derive the Euler equation

$$
x^{2} Z^{\prime \prime}+x Z^{\prime}-\left[1+\frac{1}{4} C\right] Z=0 .
$$

The expression of the general solution to equation (47), valid for $x>0$, depends on the sign of $1+C / 4$ 
1. If $1+C / 4>0$ then we get

$$
Z^{(1)}(x)=C_{1}^{(1)} x^{n}+C_{2}^{(1)} x^{-n} \quad \text { with } \quad n=\sqrt{1+C / 4}
$$

where $C_{1}^{(1)}$ et $C_{2}^{(1)}$ are constants of integration.

2. If $1+C / 4=0$ then we get

$$
Z^{(2)}(x)=C_{1}^{(2)}+C_{2}^{(2)} \ln x
$$

where $C_{1}^{(2)}$ and $C_{2}^{(2)}$ are constants of integration.

3. If $1+C / 4<0$ then we get

$$
Z^{(3)}(x)=C_{1}^{(3)} \sin (n \ln x)+C_{2}^{(3)} \cos (n \ln x) \quad \text { with } \quad n=-\sqrt{-1-C / 4}
$$

where $C_{1}^{(3)}$ and $C_{2}^{(3)}$ are constants of integration.

We now verify that solutions (48-50) satisfy the second equation (44). We find that this is true if the following constraints on the constants of integration are satisfied

$$
\left.-4 C_{1}^{(1)} C_{2}^{(1)} n^{2}=\alpha^{2} \sigma_{0} \quad\left(C_{2}^{(2)}\right)^{2}=\alpha^{2} \sigma_{0} \quad\left(\left(C_{1}^{(3)}\right)^{2}+C_{2}^{(3)}\right)^{2}\right) n^{2}=\alpha^{2} \sigma_{0} .
$$




\section{References}

[1] Kibble, T. W. B. (1976). J. Phys. A: Math. Gen. 9, 183.

[2] Vilenkin, A., and Shellard, E. P. S. (1994). Cosmic Strings and Other Topological Defects (Cambridge: Cambridge University Press).

[3] Cohen, A. G., and Kaplan, D. B. (1988). Phys. Lett. B, 215, 67.

[4] Gregory, R. (1988). Phys. Lett. B, 215, 663.

[5] Harari, D., and Sikivie, P. (1988). Phys. Rev. D, 37, 3438.

[6] Gibbons, G. W., Ortiz, M. E., and Ruiz Ruiz, F. (1989). Phys. Rev. D, 39, 1546.

[7] Larson, S. L., and Hiscock, W. A. (1997). Phys. Rev. D, 56, 3242.

[8] Gundlach, C., and Ortiz, M. E. (1990). Phys. Rev. D, 42, 2521.

[9] Barros, A., and Romero, C. (1995). J. Math. Phys., 36, 5800.

[10] Guimarães, M. E. X. (1997). Class. Quantum Grav., 14, 435.

[11] Gregory, R., and Santos, C. (1997). Phys. Rev. D, 56, 1194.

[12] Sen, A. A., Banerjee, N., and Banerjee, A. (1997). Phys. Rev. D, 56, 3706.

[13] Gibbons, G. W., Ortiz, M. E., and Ruiz Ruiz, F. (1990). Phys. Lett. B, 240, 50.

[14] Harari, D., and Polychronakos, A. P. (1990). Phys. Lett. B, 240, 55.

[15] Kamke, E. (1983). Differentialgleichungen: Lösungsmethoden und lösungen (Stuttgart: B. G. Teubner). 